Correction

\title{
Correction: Armstrong C. Unity, Continuity, Structure, and Function. The Ongoing Search for a Deeper Understanding of the Many Roles Attributed to Fascia in the Living Human Body - An Osteopathic Perspective. OBM Integrative and Complementary Medicine 2021; 6: 16
}

\author{
Colin Armstrong *
}

Osteopathic Centre, 1732 Voie Aurelienne, 13450 Grans, France; E-Mail: arm.colin@gmail.com

* Correspondence: Colin Armstrong; E-Mail: arm.colin@gmail.com

Academic Editor: Nancy Nies Byl

Special Issue: The Importance of the Fascia for Manual Osteopathic Medicine

OBM Integrative and Complementary Medicine

2021, volume 6, issue 4

doi:10.21926/obm.icm.2104035
Received: October 14, 2021

Accepted: October 14, 2021

Published: October 15, 2021

The authors wish to make the following correction to the paper [1]. Replace:

It has been suggested that the dissection of cadavers encourages "dissective thinking" [29]. There is a significant difference between the dissected, disassembled "anatomical" body as presented in anatomy textbooks, and a living, functioning organism. However, extensive knowledge of both is necessary. The creation of planes and flat surfaces by the dissector's scalpel is not an entirely accurate representation of the three-dimensional spatial organization of living matter inside a living organism, especially in terms of micro-anatomy. Sheets of fascia can be created using a scalpel during dissection to represent separate "layers" like the pages of a book. However, endoscopic exploration reveals that they do not appear to be arranged like this inside the living body but rather form an integral part of a three-dimensional, fluid-filled, fibrillar matrix [7].

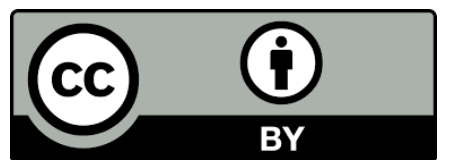

(C) 2021 by the author. This is an open access article distributed under the conditions of the Creative Commons by Attribution License, which permits unrestricted use, distribution, and reproduction in any medium or format, provided the original work is correctly cited. 


\section{Conclusions}

It is becoming increasingly apparent that the living body functions as an integrated unit, with fascia as the unifying element. The fascial system extends into every part of the body as a multidirectional web of fibers in which everything is at once connected and separated, enabling the various systems of the body to work together as a coherent, interdependent structural and functional entity. The conventional understanding of the human body as an assemblage of separate, distinct anatomical structures is being challenged by modern research that is providing a growing body of evidence of more porous and less permeable systems that extend across tissue and organ boundaries.

with:

It has been suggested that the dissection of cadavers encourages "dissective thinking" [30]. There is a significant difference between the dissected, disassembled "anatomical" body as presented in anatomy textbooks, and a living, functioning organism. However, extensive knowledge of both is necessary. The creation of planes and flat surfaces by the dissector's scalpel is not an entirely accurate representation of the three-dimensional spatial organization of living matter inside a living organism, especially in terms of micro-anatomy. Sheets of fascia can be created using a scalpel during dissection to represent separate "layers" like the pages of a book. However, endoscopic exploration reveals that they do not appear to be arranged like this inside the living body but rather form an integral part of a three-dimensional, fluid-filled, fibrillar matrix [7].

\section{Conclusions}

It is becoming increasingly apparent that the living body functions as an integrated unit, with fascia as the unifying element. The fascial system extends into every part of the body as a multidirectional web of fibers in which everything is at once connected and separated, enabling the various systems of the body to work together as a coherent, interdependent structural and functional entity. The conventional understanding of the human body as an assemblage of separate, distinct anatomical structures is being challenged by modern research that is providing a growing body of evidence of systems that extend across tissue and organ boundaries that are proving to be more porous and less impermeable than once thought.

This change has no material impact on the discussion and conclusions of the paper, but rectifies an editorial error that was not noticed during the proof reading of the paper. The author would like to apologize for any convenience caused to the readers by these changes.

\section{Competing Interests}

The authors has declared that no competing interests exist. 


\section{References}

1. Armstrong C. Unity, continuity, structure, and function. The ongoing search for a deeper understanding of the many roles attributed to fascia in the living human body - an osteopathic perspective. OBM Integr Complement Med. 2020; 5: 19. doi:10.21926/obm.icm. 2103026.

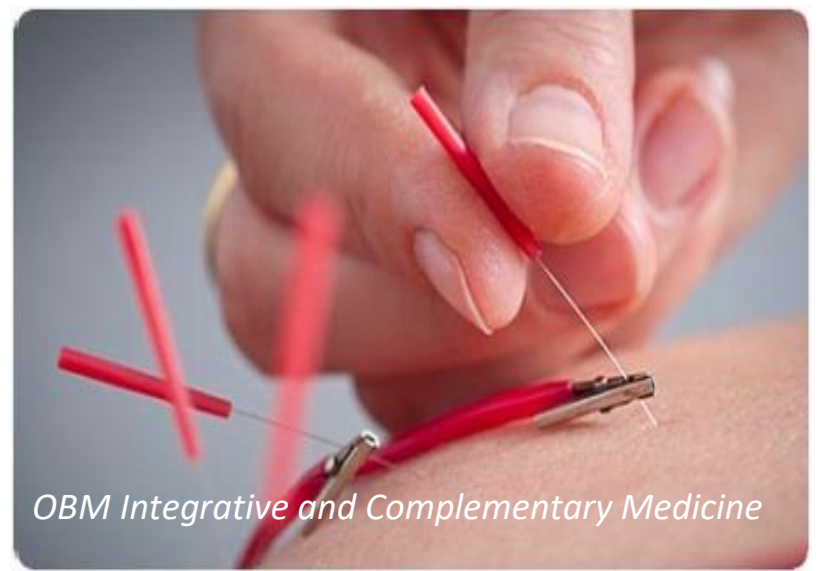

Enjoy OBM Integrative and Complementary Medicine by:

1. Submitting a manuscript

2. Joining in volunteer reviewer bank

3. Joining Editorial Board

4. Guest editing a special issue

For more details, please visit:

http://www.lidsen.com/journals/icm 